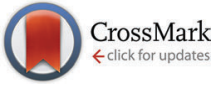

Cite this: Chem. Commun., 2016 52,300

Received 14th September 2015, Accepted 22nd October 2015

DOI: $10.1039 / \mathrm{c} 5 \mathrm{cc} 07690 \mathrm{~K}$

www.rsc.org/chemcomm

\section{Polymorphs of layered assemblies of hydrogen- bonded hexagonal networks caused by conformational frustration $\uparrow$}

\author{
Ichiro Hisaki, ${ }^{a}$ Nobuaki Ikenaka, ${ }^{a}$ Norimitsu Tohnai ${ }^{a}$ and Mikiji Miyata ${ }^{b}$
}

\begin{abstract}
Generation of four polymorphs of 2D-nCOF crystals that are formed through stacking of a hydrogen-bonded, hexagonallynetworked framework is revealed for the first time. The structural diversity is caused not from the topology or the stacking manner of the framework but from subtle structural factors such as rotational conformation and location of conformational frustration within the framework.
\end{abstract}

Functional porous materials are one of the central targets in the field of modern chemistry. ${ }^{1}$ Particularly, porous materials formed by stacking of two-dimensionally (2D) networked $\pi$-conjugated organic components have attracted much attention from the viewpoints of not only selective gas adsorbents and catalysts but also photoelectronic materials. One of the promising systems is a covalent organic framework (COF), whose facile synthesis was first demonstrated by Yaghi and co-workers, ${ }^{2}$ and subsequently, a number of excellent COFs were prepared using various $\pi$-conjugated organic building blocks. ${ }^{3}$

Structural characterization of 2D-COFs is often conducted by powder X-ray diffraction (PXRD) analysis combined with other spectroscopic methods to reveal whether porous 2D sheets are stacked in a staggered or eclipsed manner. ${ }^{4}$ However, it still remains difficult to obtain more precise structural information such as subtle slipping of the stacked layers and conformational changes of the flexible components. ${ }^{5}$ A $2 \mathrm{D}$ non-covalent organic framework (2D-nCOF), on the other hand, is a convenient model system because a single crystal suitable for $\mathrm{X}$-ray crystallographic analysis can be readily prepared by routine recrystallization from a solution, enabling more detailed

\footnotetext{
${ }^{a}$ Department of Material and Life Science, Graduate School of Engineering, Osaka University, 2-1 Yamadaoka, Suita, Osaka 565-0871, Japan.

E-mail: hisaki@mls.eng.osaka-u.ac.jp

${ }^{b}$ The Institute of Scientific and Industrial Research, Osaka University,

8-1 Mihogaoka, Ibaraki, Osaka 567-0047, Japan

$\dagger$ Electronic supplementary information (ESI) available: Synthesis, detailed crystal structures, thermal analysis, and NMR spectra. CCDC 1424086-1424091. For ESI and crystallographic data in CIF or other electronic format see DOI: $10.1039 / \mathrm{c} 5 \mathrm{cc} 07690 \mathrm{k}$
}

structural characterization. To date, the precise structures and properties of a number of 2D-nCOFs have been reported. ${ }^{6}$ We have also demonstrated the $C_{3}$-symmetric $\pi$-conjugated macrocycle, dodecadehydrotribenzo[18]annulene ([18]DBA), possessing six carboxy phenyl groups crystallized into $2 \mathrm{D}-\mathrm{nCOF},{ }^{7}$ in which a multi-porous hexagonal network (HexNet) is formed through
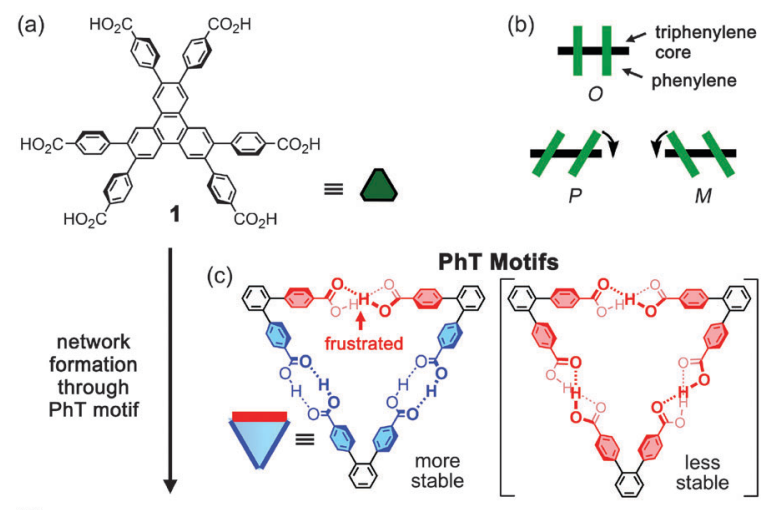

(d)

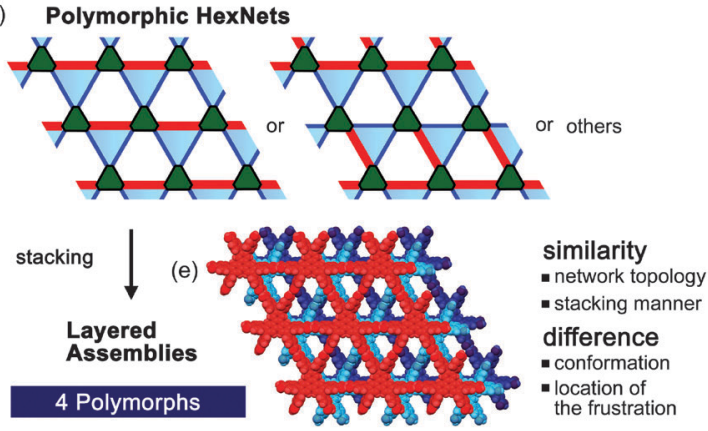

Fig. 1 Hierarchical interpretation for generation of polymorph in 2D non-covalent organic framework (2D-nCOF) crystals with hexagonal network (HexNet) of 1. (a) Molecular structure of 1 . (b) $P$ and $M$ conformations of the peripheral phenylene moieties. (c) Hydrogen-bonded phenylene triangle (PhT) motifs. (d) Polymorph of HexNets caused by location of the frustrated hydrogen bond. (e) Formation of polymorphic crystals via stacking of the HexNets. Among the obtained four polymorphic crystals, stacking manner of the HexNets is nearly the same while conformation of the peripheral groups, location of the frustration, and arrangements of guest molecules in void space are different. 
the triangular supramolecular synton, ${ }^{8}$ the so called phenylene triangle (PhT) motif. ${ }^{9}$

In this manuscript, we describe four polymorphs of 2D-nCOF composed of layered HexNets of the triphenylene derivative 1 (Fig. 1). The remarkable feature of the present polymorphic system is that HexNet sheets in the polymorphic crystals have the same topology and are laminated in nearly the same stacking manner, while the rotational conformation of the carboxy phenyl groups and location of the conformational frustration of the PhT motifs are completely different among the four crystals. To our knowledge, this is the first example for 2D-nCOF crystals to disclose as many as four polymorphic structures caused by subtle but clear structural differences such as rotational conformations.

The adjacent two peripheral phenylene groups in $\mathbf{1}$ are inclined in the same direction $(P$ or $M)$ to avoid steric repulsion between them (Fig. 1b). Therefore, the PhT motif includes at least one conformationally-frustrated hydrogen bonded carboxyl dimer (Fig. 1c), except in the case that the all phenylene groups are in the orthogonal conformation $(O)$ (Fig. 1b). To date, we have not observed a PhT motif with three frustrated dimers because of its instability, while the low symmetry PhT motif with one frustrated dimer has been frequently observed. ${ }^{7}$ Therefore, it is easy to visualize that low symmetry PhT brings the versatile location of the frustrated dimer within the HexNet sheet (Fig. 1d), and that stacking of the HexNet sheets yields polymorphs (Fig. 1e). Indeed, we obtained four polymorphs as follows.

1 was synthesized according to Scheme S1 (ESI $\dagger$ ). 2,3,6,7,10,11Hexabromotriphenylene and 4-(methoxycarbonyl)-phenylboronic acid were reacted under Suzuki-Miyaura coupling conditions, affording the hexasubstituted triphenylene derivative 2 in (a)
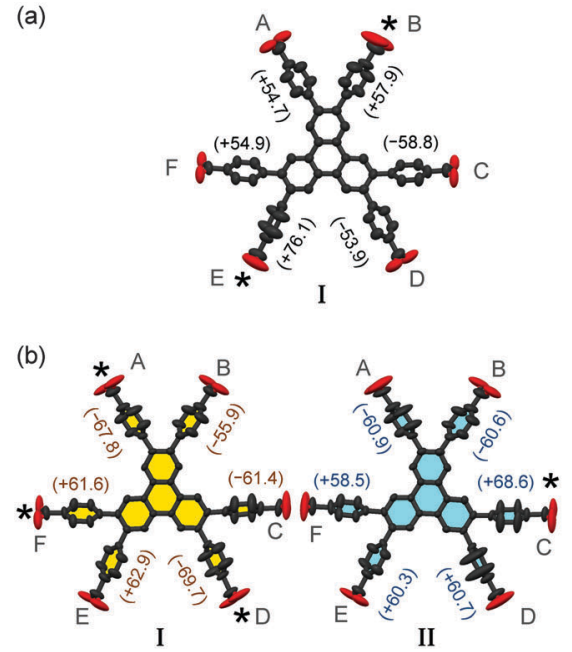

(c)

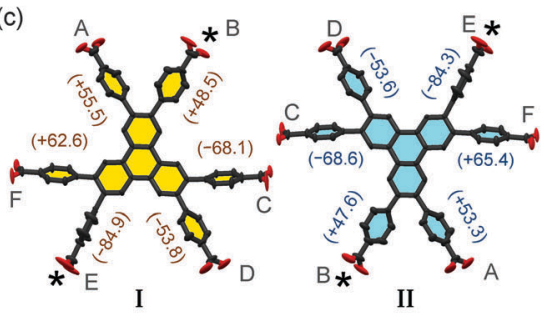

(e)

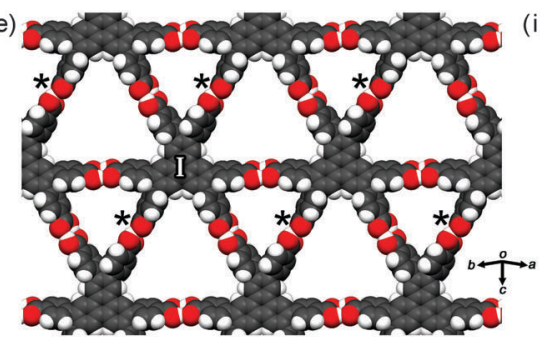

(f)

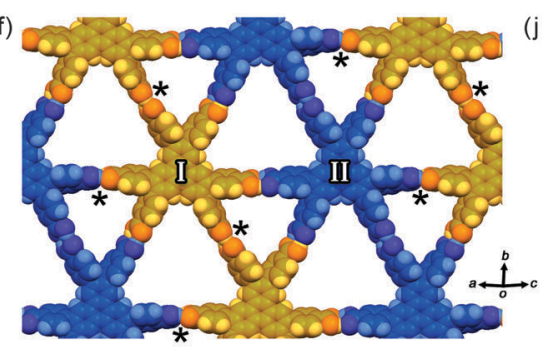

(g)

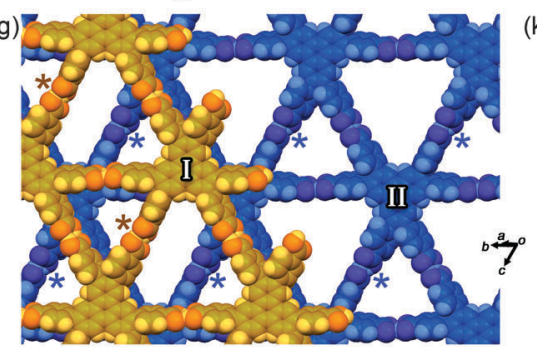

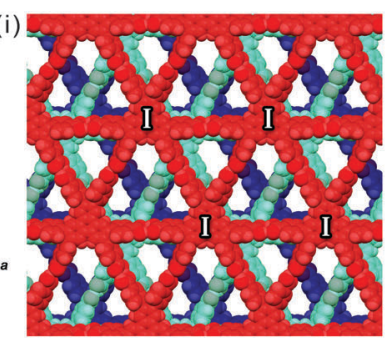
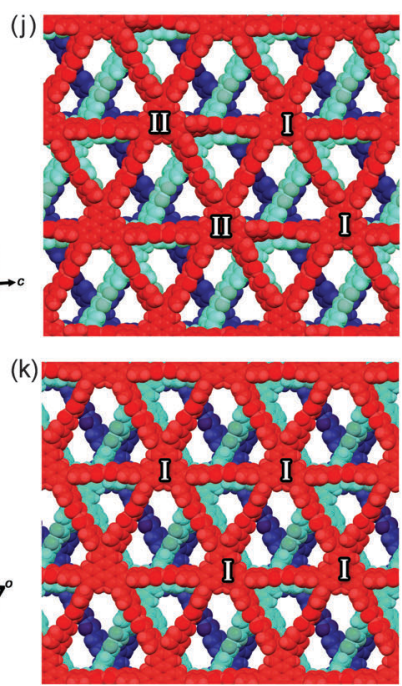

(d)

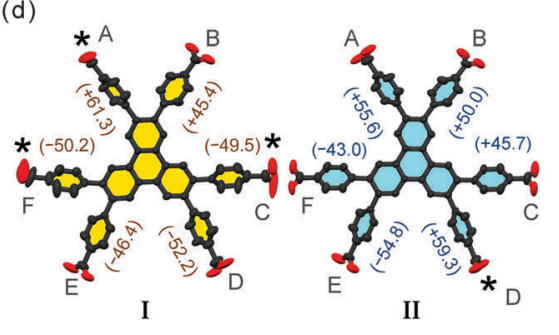

(h)

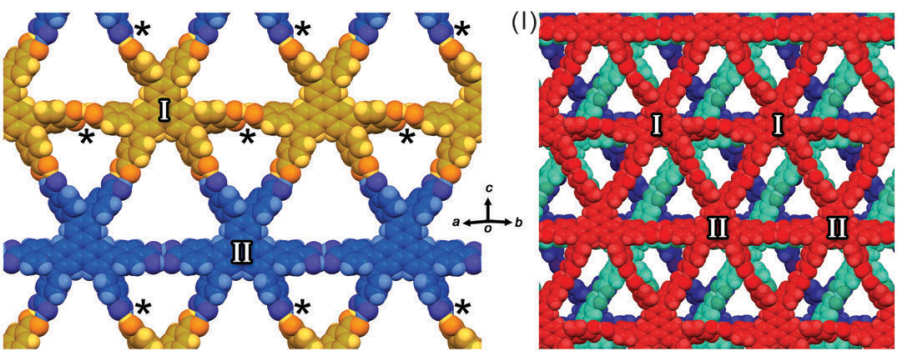

Fig. 2 Crystal structures of (a, e and i) 1-2D1, (b, f and j) 1-2D2, (c, g and k) 1-2D3, and (d, h and l) 1-2D4. Anisotropic displacement ellipsoid plot of (a) 1-2D1, (b) 1-2D2, (c) 1-2D3, and (d) 1-2D4. The ellipsoids were drawn in 75\% probability to enhance atomic displacements. 2D-HexNet structures of (e) 1-2D1, (f) 1-2D2, (g) 1-2D3 and (h) 1-2D4. Stacked three layers of (i) 1-2D1, (j) 1-2D2, (k) 1-2D3 and (l) 1-2D4. Crystallographically independent two molecules of 1 are labelled by I and II in 1-2D2, 1-2D3, and 1-2D4. Dihedral angles of the phenylene groups against the triphenylene core are presented in parentheses. An asterisk (*) points a pair of the carboxy groups possessing conformational frustration. Since the molecule II in 1-2D3 corresponds to I(A) in 1-2D1, where the symmetry code for $A:-x,-y,-z$, the sign of the dihedral angle value of II in 1-2D3 was inverted for easy compassion with others. 
96\% yield. Hydrolysis of 2 with $\mathrm{KOH}$ gave 1 in $93 \%$ yield. Recrystallization of $\mathbf{1}$ was performed by slow evaporation of a DMF and methyl benzoate solution under various temperatures. As a result, we acquired totally four kinds of perfectly-networked 2D HexNet crystals (1-2D1, 1-2D2, 1-2D3, and 1-2D4), as well as other non-hexagonally-networked crystals (see ESI $\dagger$ ). Crystals 1-2D1, 1-2D2 and 1-2D3 were obtained by recrystallization at $100{ }^{\circ} \mathrm{C}$. Crystals 1-2D1 and 1-2D2 were mainly formed concomitantly or sometimes individually depending on the crystallization batches. 1-2D3, on the other hand, was rarely formed in many batches and quality of its crystal data have not been enough yet. At this moment, we have not found a condition that yields each polymorph selectively. The crystal 1-2D4 was obtained by rapidly cooling from $100{ }^{\circ} \mathrm{C}$ to $10{ }^{\circ} \mathrm{C}$ and maintaining at that temperature for a few days.

X-ray crystal structures of the fully-networked HexNet crystals (1-2D1, 1-2D2, 1-2D3, and 1-2D4) are shown in Fig. 2. In their structures, all carboxy groups of $\mathbf{1}$ form self-complementary hydrogen bonded dimers and subsequently form the PhT motif to achieve porous HexNet structures. The anisotropic displacement ellipsoids of the carboxy groups are more elongated than those of the triphenylene core because of rotational flexibility of the groups; in particular, those of the frustrated ones are significant. The HexNet involves two types of triangle pores: one with diameter of $9.7 \AA$ originated from the PhT motif and the other with a wider diameter of $14.2 \AA$ A 1-2D1 includes a crystallographically independent molecule of 1, while 1-2D2, 1-2D3 and 1-2D4 do two molecules (I and II). The HexNet sheet in 1-2D2 and 1-2D4 is composed of alternately aligned I and II molecules (Fig. $2 \mathrm{f}$ and h), while in the case of 1-2D3, molecules I and II individually form the corresponding HexNet sheets (Fig. 2g). These four crystals are composed of conformers of 1 with different rotational angles of the phenylene and carboxy groups: $P M P$ for 1-2D1, $M M P$ and $M P P$ for 1-2D2, $P M P$ and $P M P$ for 1-2D3, and $P M M$ and $P P M$ for 1-2D4 (Fig. 2a-d and Table 1). The diverse conformation, in addition to the number of

Table 1 Dihedral angle between the phenylene ring and triphenylene skeleton ${ }^{a}$

\begin{tabular}{lcccc}
\hline & 1-2D1 & $\mathbf{1 - 2 D 2}$ & $\mathbf{1 - 2 D 3}$ & $\mathbf{1 - 2 D 4}$ \\
\hline $\mathrm{A}(\mathrm{I})$ & +54.7 & $-67.8^{*}$ & +55.5 & $+61.29^{*}$ \\
$\mathrm{~A}(\mathrm{II})$ & & -60.9 & +53.3 & +55.58 \\
$\mathrm{~B}(\mathrm{I})$ & $+57.9^{*}$ & -55.9 & $+48.5^{*}$ & +45.36 \\
$\mathrm{~B}(\mathrm{II})$ & & -60.6 & $+47.6^{*}$ & +49.98 \\
$\mathrm{C}(\mathrm{I})$ & -58.8 & -61.4 & -68.1 & $-49.45^{*}$ \\
$\mathrm{C}(\mathrm{II})$ & & $+68.6^{*}$ & -68.6 & +45.72 \\
$\mathrm{D}(\mathrm{I})$ & -53.9 & $-69.7^{*}$ & -53.8 & -52.21 \\
$\mathrm{D}(\mathrm{II})$ & & +60.7 & -53.6 & $+59.26^{*}$ \\
$\mathrm{E}(\mathrm{I})$ & $+76.1^{*}$ & +62.9 & $-84.9^{*}$ & -46.36 \\
$\mathrm{E}(\mathrm{II})$ & & +60.3 & $-84.3^{*}$ & -54.79 \\
$\mathrm{~F}(\mathrm{I})$ & +54.9 & $+61.6^{*}$ & +62.6 & $-50.16^{*}$ \\
$\mathrm{~F}(\mathrm{II})$ & & +58.5 & +65.4 & -42.95 \\
Conformer (I) & $P M P$ & $M M P$ & $P M P^{b}$ & $P M M$ \\
Conformer (II) & & $M P P$ & $P M P^{b}$ & $P P M$
\end{tabular}

${ }^{a}$ An asterisk ( ${ }^{*}$ ) denotes that the conformational frustration is located in the corresponding peripheral group. ${ }^{b}$ Phenylene ring at the $E$ position has nearly orthogonal and the sign of dihedral angle is opposite to that at $F$ position. However, the conformation at the $E-F$ bay area can denote as $P$, considered the sign of the dihedral angle at $F$ position. the crystallographically independent molecules $\left(Z^{\prime}=1\right.$ or 2$)$, results in the versatile location of conformational frustration of the hydrogen-bonded dimer in a HexNet sheet. The frustrated dimer is marked with asterisks in Fig. 2e-h. The HexNet sheet was slip-stacked without interpenetration to give a 3D framework. The layers are laminated in an $\mathrm{AB}$ manner for 1-2D1, 1-2D2, and 1-2D4, and in an AA'BB' manner for 1-2D3. Fig. 2i-l show selected three layers of the HexNet viewed from directly above.

To our surprise, the stacking manner of the HexNets in these four crystals is nearly identical, despite their versatile conformation: Averaged inter-layer distances of 1-2D1, 1-2D2, 1-2D3, and 1-2D4 are $4.72 \AA, 4.76 \AA$, $4.72 \AA$, and $4.41 \AA$, respectively, and distances between centroids of the adjacent two triphenylene cores in the crystals are 8.07-8.13 $\AA$, 7.20-8.05 $\AA$, 7.88-8.89 $\AA$, and 7.26$7.37 \AA$, respectively. The PXRD patterns simulated from the guestexcluded HexNet frameworks of 1-2D1, 1-2D2, 1-2D3, and 1-2D4 also indicate that they have quite similar periodic profiles (Fig. S2, $\mathrm{ESI} \dagger$ ). The more detailed stacking manner of the HexNets is shown in Fig. 3a, taking 1-2D1's structure as a representative example. The rhombic frame, a motif of the HexNet, stacks with another rhombic frame lying on the neighboring sheet in an inverted manner so that these frames are in contact with each other. Intermolecular interactions to make the rhombic frame stacked in a unique manner are self-complementary $\mathrm{CH} / \mathrm{O}$ contacts between hydrogen atoms of the phenylene groups and oxygen atoms in the carboxy groups, as well as $\mathrm{CH} / \pi$ contacts between the phenylene groups and the triphenylene plane (Fig. $3 \mathrm{~b}$ ). $\pi / \pi$ interaction between the triphenylene parts, on the other hand, is not observed because of steric hindrance of the phenylene groups: the averaged interplanar distance is $4.5 \AA$.
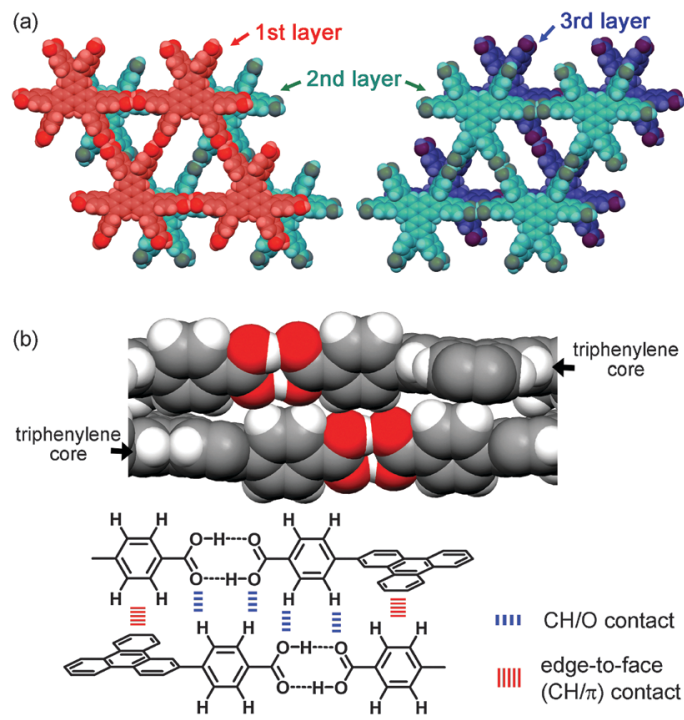

Fig. 3 (a) Stacking manners of the adjacent two rhombic motifs in 1-2D1. (b) Leading intermolecular interactions observed between the adjacent HexNet sheets of 1-2D1. CH/O interactions between hydrogen atoms of the phenylene rings and oxygen atoms of the carboxy groups (blue dash line). Edge-to-face contacts ( $\mathrm{CH} / \pi$ interactions) between hydrogen bonds of the phenylene rings and $\pi$-conjugated plane of the triphenylene ring (red dash line). The other three polymorphs also have the same intermolecular interactions. 
The solvent accessible volume and cavity ratio for the unit cell of the layered HexNet crystals are $2241.0 \AA^{3}(54.4 \%)$ for 1-2D1, $4480.4 \AA^{3}$ (54.1\%) for 1-2D2, $4466.8 \AA^{3}$ (54.4\%) for 1-2D3, and $3827.8 \AA^{3}$ (50.0\%) for 1-2D4. ${ }^{10}$ The methyl benzoate molecules accommodated in the void space are highly disordered and many of them were not capable of being refined crystallographically. $\S$ Because of guest molecule disorder, the completeness of the crystallographic data is relatively low for all crystals. The numbers of the methyl benzoate molecules refined are 1, 0 , and 2 for 1-2D1, 1-2D2, and 1-2D3, respectively, and 5 for 1-2D4. Since the former three crystals were formed at $100{ }^{\circ} \mathrm{C}$, such high temperature conditions enhanced thermal molecular motion, resulting in significant disorder of the guest molecules. However, it is noteworthy that the position and orientation of the refined guest molecules in the void space are different among the polymorphs. (Fig. S3, ESI $\dagger$ ). This implies that even when the stacking manner of the HexNet sheet is nearly the same, the inclusion behavior of the guest molecules is strongly influenced by conformational differences (i.e. dihedral angle and location of the conformational frustration).

In conclusion, we reported for the first time, four polymorphs of 2D-nCOF crystals composed of layered HexNets. The structural diversity was brought, not from the topology or the stacking manner of HexNets, but from more subtle structural factors such as rotational conformation and location of the conformational frustration in the PhT motifs. This kind of structural diversity has been difficult for discussing in conventional porous 2D frameworks, such as 2D-COFs. Thus, precise characterization of the present polymorphic HexNet crystals can provide a new insight into porous 2D-COFs.

This work was supported by Grant-in-Aid for Scientific Research (C) (T15K04591) and for Scientific Research on Innovative Areas: $\pi$-System Figuration (15H00998) from MEXT Japan. Crystallographic data were collected using synchrotron radiation at the BL38B1 in the SPring-8 with approval from JASRI (proposal No. 2014B1168 and 2015A1174).

\section{Notes and references}

\$ Crystal data for 1-2D1: $\left(\mathrm{C}_{60} \mathrm{H}_{36} \mathrm{O}_{12}\right) \cdot\left(\mathrm{C}_{8} \mathrm{H}_{8} \mathrm{O}_{2}\right), F_{\mathrm{w}}=1085.09, a=$ 14.5303(5) $\AA, b=14.9461(6) \AA, c=20.2579(8) \AA, \alpha=102.3554(18)^{\circ}, \beta=$ 101.5441(16) ${ }^{\circ}, \gamma=99.0611(17)^{\circ}, V=4117.4(3) \AA^{3}, T=153 \mathrm{~K}$, triclinic, space group $P \overline{1}, Z=2,46186$ collected, 14502 unique $\left(R_{\text {int }}=0.0798\right)$ reflections, the final $R_{1}$ and $\mathrm{w} R_{2}$ values $0.091(I>2.0 \sigma(I))$ and 0.326 (all data), respectively. Crystal data for 1-2D2: $2\left(\mathrm{C}_{60} \mathrm{H}_{36} \mathrm{O}_{12}\right), F_{\mathrm{W}}=1897.87$, $a=15.4431(3) \AA, b=20.9868(4) \AA, c=28.0527(6) \AA, \alpha=103.3620(13)^{\circ}$, $\beta=98.9655(11)^{\circ}, \gamma=105.8050(10)^{\circ}, V=8275.7(3) \AA^{3}, T=273 \mathrm{~K}$, triclinic, space group $P \overline{1}, Z=2,87842$ collected, 22359 unique $\left(R_{\text {int }}=0.103\right)$ reflections, the final $R_{1}$ and $w R_{2}$ values $0.127(I>2.0 \sigma(I))$ and 0.389 (all data), respectively. Crystal data for 1-2D3: $2\left(\mathrm{C}_{60} \mathrm{H}_{36} \mathrm{O}_{12}\right) \cdot 2\left(\mathrm{C}_{8} \mathrm{H}_{8} \mathrm{O}_{2}\right)$, $F_{\mathrm{w}}=2170.17, a=19.1278(2) \AA, b=22.4030(2) \AA, c=22.3984(2) \AA, \alpha=$ $60.0460(6)^{\circ}, \beta=87.4093(5)^{\circ}, \gamma=81.0545(4)^{\circ}, V=8209.05(14) \AA^{3}, T=93 \mathrm{~K}$, triclinic, space group $P \overline{1}, Z=2,44304$ collected, 26851 unique $\left(R_{\mathrm{int}}=\right.$ $0.045)$ reflections, the final $R_{1}$ and $\mathrm{w} R_{2}$ values $0.125(I>2.0 \sigma(I))$ and 0.445 (all data), respectively. Crystal data for 1-2D4: $2\left(\mathrm{C}_{60} \mathrm{H}_{36} \mathrm{O}_{12}\right)$. $5\left(\mathrm{C}_{8} \mathrm{H}_{8} \mathrm{O}_{2}\right), F_{\mathrm{W}}=2578.62, a=12.8218(9) \AA, \quad b=15.8756(11) \AA, c=$ $38.6736(18) \AA, \alpha=93.295(3)^{\circ}, \beta=93.991(3)^{\circ}, \gamma=102.380(3)^{\circ}, V=$ 7649.3(8) $\AA^{3}, T=213 \mathrm{~K}$, triclinic, space group $P \overline{1}, Z=2,42603$ collected, 22464 unique $\left(R_{\mathrm{int}}=0.058\right)$ reflections, the final $R_{1}$ and $\mathrm{w} R_{2}$ values $0.108(I>2.0 \sigma(I))$ and 0.360 (all data), respectively. CCDC numbers: CCDC1424089(1-2D1), 1424090(1-2D2), 1424091(1-2D3) and 1424086(1-2D4).

$\S{ }^{1} \mathrm{H}$ NMR spectrum of the crystalline bulk containing 1-2D1, 1-2D2 and 1-2D3 dissolved in DMSO- $d_{6}$ indicates that no DMF but methyl benzoate molecules are included in the crystals with a 1:6 molar ratio of 1 and methyl benzoate (Fig. S4, ESI $\dagger$ ). Moreover, thermal gravimetric analysis curve reaches a bottom at $240{ }^{\circ} \mathrm{C}$ with a weight loss of $47 \%$, which is in good agreement with a theoretical value $(46.3 \%)$ assuming a $1: 6$ ratio of $\mathbf{1}$ and methyl benzoate ratio (Fig. S5, ESI $\dagger$ ). These results are consistent with the size of cavity volume in the crystals.

1 A. G. Skater and A. I. Cooper, Science, 2015, 348, aaa8075.

2 A. P. Côté, A. I. Benin, N. W. Ockwig, M. O'Keeffe, A. J. Matzger and O. M. Yaghi, Science, 2005, 310, 1166.

3 (a) X. Feng, X. Ding and D. Jiang, Chem. Soc. Rev., 2012, 41, 6010; (b) S.-Y. Ding and W. Wang, Chem. Soc. Rev., 2013, 42, 548.

4 Recent examples, see; (a) M. Dogru, M. Handloser, F. Auras, T. Kunz, D. Medina, A. Hartschuh, P. Knochel and T. Bein, Angew. Chem., Int. Ed., 2013, 52, 2920; (b) H. Yang, Y. Du, S. Wan, G. D. Trahan, Y. Jin and W. Zhang, Chem. Phys., 2015, 6, 4049; (c) L. A. Baldwin, J. W. Crowe, M. D. Shannon, C. P. Jaroniec and P. J. McGrier, Chem. Mater., 2015, 27, 6169.

5 Detailed slipping manner of COF layers was investigated, see: B. Lukose, A. Kuc and T. Heine, Chem. - Eur. J., 2011, 17, 2388.

6 (a) D. J. Duchamp and R. Marsh, Acta Crystallogr., Sect. B: Struct. Crystallogr. Cryst. Chem., 1969, 25, 5; (b) F. H. Herbstein, M. Kapon and G. M. Reisner, J. Inclusion Phenom., 1987, 5, 211; (c) R. E. Melendez, C. V. K. Sharma, M. J. Zaworotko, C. Bauer and R. D. Rogers, Angew. Chem., Int. Ed. Engl., 1996, 35, 2213; (d) A. R. A. Palmans, J. A. J. M. Vekemans, H. Kooijman, A. L. Spek and E. W. Meijer, Chem. Commun., 1997, 2247; (e) P. Sozzani, A. Comotti, R. Simonutti, T. Meersmann, J. W. Logan and A. Pines, Angew. Chem., Int. Ed., 2000, 39, 2695; $(f)$ K. Kobayashi, A. Sato, S. Sakamoto and K. Yamaguchi, J. Am. Chem. Soc., 2003, 125, 3035; $(g)$ P. Sozzani, S. Bracco, A. Comotti, L. Ferretti and R. Simonutti, Angew. Chem., Int. $E d ., 2005,44,1816 ;(h)$ K. E. Maly, E. Gagnon, T. Maris and J. D. Wuest, J. Am. Chem. Soc., 2007, 129, 4306; (i) Y.-B. Men, J. Sun, Z.-T. Huang and Q.-Y. Zheng, Angew. Chem., Int. Ed., 2009, 48, 2873; (j) X.-Z. Luo, X.-J. Jia, J.-H. Deng, J.-L. Zhong, H.-J. Liu, K.-J. Wang and D.-C. Zhong, J. Am. Chem. Soc., 2013, 135, 11684; (k) T.-H. Chen, I. Popov, W. Kaveevivitchai, Y.-C. Chuang, Y.-S. Chen, O. Daugulis, A. J. Jacobson and O. Š. Miljanić, Nat. Commun., 2014, 5, 5131.

7 I. Hisaki, S. Nakagawa, N. Tohnai and M. Miyata, Angew. Chem., Int. Ed., 2015, 54, 3008.

8 For supramolecular synthon, see; G. R. Desiraju, Angew. Chem., Int. Ed. Engl., 1995, 34, 2311.

9 The first report of the PhT motif; K. Kobayashi, T. Shirasaka, E. Horn and N. Furukawa, Tetrahedron Lett., 2000, 41, 89.

10 Calculation was conducted by PLATON software with the following atomic radii: $1.20 \AA(\mathrm{H}), 1.70 \AA(\mathrm{C}), 1.52 \AA(\mathrm{O})$. The probe radius was $1.2 \AA$. 